\title{
Genome wide linkage disequilibrium and genetic structure in Sicilian dairy sheep breeds
}

\author{
Salvatore Mastrangelo ${ }^{*}$, Rosalia Di Gerlando, Marco Tolone, Lina Tortorici, Maria Teresa Sardina, \\ Baldassare Portolano and International Sheep Genomics Consortium
}

\begin{abstract}
Background: The recent availability of sheep genome-wide SNP panels allows providing background information concerning genome structure in domestic animals. The aim of this work was to investigate the patterns of linkage disequilibrium (LD), the genetic diversity and population structure in Valle del Belice, Comisana, and Pinzirita dairy sheep breeds using the Illumina Ovine SNP50K Genotyping array.

Results: Average $r^{2}$ between adjacent SNPs across all chromosomes was $0.155 \pm 0.204$ for Valle del Belice, $0.156 \pm 0.208$ for Comisana, and $0.128 \pm 0.188$ for Pinzirita breeds, and some variations in LD value across chromosomes were observed, in particular for Valle del Belice and Comisana breeds. Average values of $r^{2}$ estimated for all pairwise combinations of SNPs pooled over all autosomes were $0.058 \pm 0.023$ for Valle del Belice, $0.056 \pm 0.021$ for Comisana, and $0.037 \pm 0.017$ for Pinzirita breeds. The LD declined as a function of distance and average $r^{2}$ was lower than the values observed in other sheep breeds. Consistency of results among the several used approaches (Principal component analysis, Bayesian clustering, $F_{\mathrm{ST}}$, Neighbor networks) showed that while Valle del Belice and Pinzirita breeds formed a unique cluster, Comisana breed showed the presence of substructure. In Valle del Belice breed, the high level of genetic differentiation within breed, the heterogeneous cluster in Admixture analysis, but at the same time the highest inbreeding coefficient, suggested that the breed had a wide genetic base with inbred individuals belonging to the same flock. The Sicilian breeds were characterized by low genetic differentiation and high level of admixture. Pinzirita breed displayed the highest genetic diversity $\left(\mathrm{He}, \mathrm{N}_{\mathrm{e}}\right)$ whereas the lowest value was found in Valle del Belice breed.

Conclusions: This study has reported for the first time estimates of LD and genetic diversity from a genomewide perspective in Sicilian dairy sheep breeds. Our results indicate that breeds formed non-overlapping clusters and are clearly separated populations and that Comisana sheep breed does not constitute a homogenous population. The information generated from this study has important implications for the design and applications of association studies as well as for development of conservation and/or selection breeding programs.
\end{abstract}

Keywords: OvineSNP50K BeadChip, Sicilian sheep breeds, Linkage disequilibrium, Genome structure

\section{Background}

The application of recently developed genomic technology, such as high-density single nucleotide polymorphism (SNP) arrays, has great potential to increase our understanding on the genetic architecture of complex traits, to improve selection efficiency in domestic animals through genomic selection [1], and to conduct association studies [2]. However, to optimally plan whole-genome

\footnotetext{
* Correspondence: salvatore.mastrangelo@unipa.it

Dipartimento Scienze Agrarie e Forestali, Università degli Studi di Palermo, Viale delle Scienze, 90128 Palermo, Italy
}

(c) 2014 Mastrangelo et al.; licensee BioMed Central Ltd. This is an Open Access article distributed under the terms of the Creative Commons Attribution License (http://creativecommons.org/licenses/by/4.0), which permits unrestricted use, distribution, and reproduction in any medium, provided the original work is properly credited. The Creative Commons Public Domain Dedication waiver (http://creativecommons.org/publicdomain/zero/1.0/) applies to the data made available in this article, unless otherwise stated.

association studies, it is crucial to know the extent of linkage disequilibrium (LD), the non-random association of alleles at different loci in the genome. In fact, the extent of LD is often used to determine the optimal number of markers required for fine mapping of quantitative trait loci (QTL) [3], for genomic selection [4], and to understand the evolutionary history of the populations [5]. With this in mind, it is important to quantify the extent of LD within different breeds as this is likely to have an impact on the success of gene mapping experiments [6]. Knowledge concerning the extent of genetic diversity, as the level of inbreeding and population structure is critical for 
each of these applications [7]. Moreover, effective population size $\left(\mathrm{N}_{\mathrm{e}}\right)$ is an important parameter for the assessment of genetic diversity within a livestock population and its development over time. If pedigree information is not available, LD analysis might offer an alternative perspective for the estimation of $\mathrm{N}_{\mathrm{e}}$ [5]. In local breeds, maintaining genetic variability is an important requirement for animal breeding strategies; this guarantees selection response to productive and adaptive traits improvement, to cope with new environmental conditions, changes in market demands, husbandry practices and disease challenges [8]. Currently, with availability of high-density SNP arrays, genetic diversity can be estimated accurately in the absence of pedigree information [9].

In Sicily, dairy sheep production represents an important resource for the economy of hilly and mountain areas, in which other economic activities are limited [10]. Nowadays, three native dairy sheep breeds are reared in Sicily: Valle del Belice, Comisana and Pinzirita. These breeds present differences both in morphology and production traits, showing excellent adaptability to local environments. The aim of this work was to investigate these breeds from a genetic perspective, including the analysis of: i) genome-wide levels of LD, ii) genetic diversity and population structure using high-density genotyping arrays.

\section{Results and discussion}

In the present study, the Illumina OvineSNP50K Genotyping BeadChip was used to characterize LD and to analyze genetic diversity and population structure in Sicilian dairy sheep breeds.

Out of a total of 54,241 SNPs genotyped, 378 were unmapped and 1,450 were located on sex chromosomes. Thus, 52,413 SNPs mapped onto 26 sheep autosomes were used, and after filtering (see Methods), the final number of samples and SNPs were 71 and 44,365 for Valle del Belice, 71 and 44,540 for Comisana, and 77 and 45,451 for Pinzirita sheep breeds. The distribution of SNPs per chromosome and breed was reported in Additional file 1: Table S1.

\section{Linkage Disequilibrium (LD)}

The extent of LD was first evaluated for each adjacent SNP pairs. The $r^{2}$ was used as measure of LD, because is the most suitable measure of LD for biallelic markers [11] and to avoid the influence of small sample size [12]. The average distances between adjacent SNP pairs for the whole autosomal genome were about $60 \mathrm{~kb}$ for Valle del Belice and Comisana, and $59 \mathrm{~kb}$ for Pinzirita sheep breeds (Table 1). The $r^{2}$ ranged from $0.133 \pm 0.187$ for OAR14 to $0.182 \pm 0.229$ for OAR2 in Valle del Belice, from $0.134 \pm 0.188$ for OAR14 to $0.189 \pm 0.237$ for OAR2 in Comisana, whereas the lowest values among chromosomes were observed in Pinzirita, where $r^{2}$ ranged from $0.107 \pm 0.164$ for OAR23 to $0.148 \pm 0.208$ for OAR2 (Table 1). Mean values of $r^{2}$ estimated for all pairwise SNPs combinations pooled over all autosomes were $0.058 \pm 0.023$ for Valle del Belice, $0.056 \pm 0.021$ for Comisana, and $0.037 \pm 0.017$ for Pinzirita sheep breeds. In order to examine the decay of LD with physical distance, SNP pairs on autosomes were sorted into bins based on their inter-marker distance and average values of $r^{2}$ were calculated for each bin. Pairwise $r^{2}$ values were also averaged over all autosomes and plotted as a function of genomic distance between markers. Levels of pairwise LD decreased with increasing distance between SNPs, as reported in Figure 1 and Additional file 2: Table $\mathrm{S} 2$. For SNPs up to $50 \mathrm{~kb}$ apart the average $r^{2}$ was 0.183 , 0.181 , and 0.154 , for SNPs separated by $200-500 \mathrm{~kb}$ the average $r^{2}$ was $0.074,0.067$, and 0.041, and when SNPs were separated by more than $2,000 \mathrm{~kb}$ the average $r^{2}$ was 0.041, 0.042 and 0.029 in Valle del Belice, Comisana, and Pinzirita sheep breeds, respectively. These results could be attributed to recombination rate varying between and within chromosomes, differences in chromosome length, heterozygosity, genetic drift, and effect of selection [13]. Effect of selection on LD is dependent upon direction, intensity, duration, and consistency of selection over time. In fact, Pinzirita breed is not subject to breeding programs, while Comisana and Valle del Belice breeds are characterized by low selection pressure. The comparison of LD levels obtained in different studies is not straightforward, because of differences in several factors such as sample size, type of LD measures ( $D^{\prime}$ or $r^{2}$ ), marker types (microsatellite or SNP), marker density and distribution, and population demography [13]. Moreover, so far, results of the extent of LD have been reported for wide-spread and important sheep breeds, and there is little knowledge about the degree of genome-wide LD in local sheep breeds. García-Gámez et al. [1] in a study on LD in Spanish Churra sheep breed reported an average $r^{2}$ estimated for all pairwise combinations per chromosomes that ranged from 0.006 in OAR1 to 0.015 in OAR20, and an average $r^{2}$ of 0.061 for SNPs separated by 200-500 kb, in agreement with our results. Usai et al. [14] in a study of LD in a sample of Sarda rams showed higher value with an average $r^{2}$ over $1,000 \mathrm{~kb}$ of 0.072 . Previous studies in five populations of domestic sheep based on microsatellite markers [6] and in wild sheep based on dense panel of SNPs [15] showed LD extends over long distance $\left(r^{2}=0.192 \pm 0.131\right.$ for markers separated by 5-10 cM, and high levels of LD observed over $4 \mathrm{Mb}$, respectively). Levels of $r^{2}$ for adjacent SNP pairs and all pairwise combinations of SNPs in Sicilian sheep breeds were lower than values observed in other livestock species such as pig [16,17], cattle [13,18], and horse $[19,20]$. These differences can be explained considering the intensive artificial selection to which commercial 
Table 1 Average space (bp), Linkage Disequilibrium $\left(r^{2}\right)$ and standard deviation (s.d.) between adjacent single nucleotide polymorphisms (SNPs) on each chromosome (OAR) in the Sicilian sheep breeds

\begin{tabular}{|c|c|c|c|c|c|c|}
\hline & VDB & & COM & & PIN & \\
\hline OAR & Average space & $r^{2} \pm$ s.d. & Average space & $r^{2} \pm$ s.d. & Average space & $r^{2} \pm$ s.d. \\
\hline 1 & 60,381 & $0.162 \pm 0.205$ & 60,211 & $0.161 \pm 0.213$ & 58,874 & $0.132 \pm 0.190$ \\
\hline 2 & 56,594 & $0.182 \pm 0.229$ & 57,023 & $0.189 \pm 0.237$ & 55,298 & $0.148 \pm 0.208$ \\
\hline 3 & 57,120 & $0.170 \pm 0.217$ & 56,744 & $0.172 \pm 0.224$ & 55,389 & $0.140 \pm 0.198$ \\
\hline 4 & 55,435 & $0.171 \pm 0.219$ & 54,484 & $0.164 \pm 0.216$ & 53,770 & $0.139 \pm 0.200$ \\
\hline 5 & 57,306 & $0.150 \pm 0.199$ & 57,292 & $0.154 \pm 0.205$ & 55,749 & $0.127 \pm 0.188$ \\
\hline 6 & 57,242 & $0.158 \pm 0.204$ & 57,138 & $0.165 \pm 0.216$ & 55,381 & $0.131 \pm 0.188$ \\
\hline 7 & 56,080 & $0.151 \pm 0.200$ & 56,256 & $0.158 \pm 0.209$ & 55,645 & $0.137 \pm 0.194$ \\
\hline 8 & 54,964 & $0.164 \pm 0.211$ & 54,444 & $0.155 \pm 0.206$ & 54,142 & $0.133 \pm 0.192$ \\
\hline 9 & 55,391 & $0.167 \pm 0.218$ & 54,938 & $0.170 \pm 0.222$ & 53,755 & $0.137 \pm 0.196$ \\
\hline 10 & 57,388 & $0.168 \pm 0.224$ & 56,578 & $0.179 \pm 0.230$ & 56,003 & $0.148 \pm 0.217$ \\
\hline 11 & 68,228 & $0.155 \pm 0.198$ & 66,998 & $0.155 \pm 0.208$ & 65,042 & $0.118 \pm 0.177$ \\
\hline 12 & 58,985 & $0.165 \pm 0.210$ & 58,205 & $0.157 \pm 0.207$ & 57,224 & $0.131 \pm 0.190$ \\
\hline 13 & 61,411 & $0.151 \pm 0.204$ & 60,698 & $0.170 \pm 0.219$ & 60,245 & $0.133 \pm 0.197$ \\
\hline 14 & 67,767 & $0.133 \pm 0.187$ & 67,759 & $0.134 \pm 0.188$ & 67,623 & $0.114 \pm 0.182$ \\
\hline 15 & 61,225 & $0.149 \pm 0.202$ & 61,225 & $0.153 \pm 0.209$ & 59,525 & $0.129 \pm 0.189$ \\
\hline 16 & 57,537 & $0.149 \pm 0.199$ & 58,498 & $0.157 \pm 0.212$ & 56,483 & $0.124 \pm 0.184$ \\
\hline 17 & 65,423 & $0.155 \pm 0.208$ & 64,034 & $0.152 \pm 0.208$ & 62,553 & $0.125 \pm 0.191$ \\
\hline 18 & 60,008 & $0.149 \pm 0.200$ & 59,676 & $0.156 \pm 0.205$ & 59,039 & $0.128 \pm 0.191$ \\
\hline 19 & 60,499 & $0.156 \pm 0.213$ & 61,012 & $0.167 \pm 0.217$ & 59,829 & $0.139 \pm 0.201$ \\
\hline 20 & 57,307 & $0.150 \pm 0.197$ & 57,482 & $0.138 \pm 0.187$ & 56,644 & $0.117 \pm 0.168$ \\
\hline 21 & 69,546 & $0.152 \pm 0.206$ & 68,146 & $0.151 \pm 0.205$ & 68,551 & $0.124 \pm 0.175$ \\
\hline 22 & 57,485 & $0.154 \pm 0.204$ & 57,852 & $0.155 \pm 0.209$ & 56,128 & $0.125 \pm 0.192$ \\
\hline 23 & 69,395 & $0.156 \pm 0.193$ & 68,358 & $0.140 \pm 0.189$ & 67,233 & $0.107 \pm 0.164$ \\
\hline 24 & 71,118 & $0.134 \pm 0.184$ & 71,232 & $0.134 \pm 0.187$ & 70,438 & $0.108 \pm 0.168$ \\
\hline 25 & 55,503 & $0.141 \pm 0.190$ & 56,750 & $0.142 \pm 0.202$ & 54,932 & $0.116 \pm 0.183$ \\
\hline 26 & 61,598 & $0.143 \pm 0.190$ & 62,022 & $0.137 \pm 0.178$ & 59,862 & $0.116 \pm 0.171$ \\
\hline mean & 60,421 & $0.155 \pm 0.204$ & 60,194 & $0.156 \pm 0.208$ & 59,062 & $0.128 \pm 0.188$ \\
\hline
\end{tabular}

Valle del Belice (VDB), Comisana (COM) and Pinzirita (PIN) sheep breeds.

animal breeding populations (pig, cattle and horse) have been subjected for many generations and the ensuing reduction in effective population size. In fact, these species share a similar mating system using popular sires, whereas in ovine species, and in particular in Sicilian farming system, natural mating is the common practice. The decay of LD in a genome determines the power of QTL detection in association mapping studies and helps to determine the number of markers required for successful association mapping and genomic selection. Meuwissen et al. [4], in a simulation to predict genomic breeding values from dense markers across the whole genome with accuracies up to 0.85 , found a required $r^{2}$ level of 0.2. Qanbari et al. [13] considered $r^{2}$ threshold of 0.25 as a useful LD value for association studies. In fact, species with extensive LD will require fewer markers than those with low levels of LD.
Therefore, these results support the need to use more dense SNP panels for high power association mapping and genomic selection efficiency in future breeding programs for Sicilian dairy sheep breeds, and in particular for Pinzirita breed.

\section{Genetic diversity and population structure}

Principal Component Analysis (PCA), Bayesian modelbased clustering algorithm, calculation of $F_{\mathrm{ST}}$ and Neighbor network were used to visualize and explore the genetic relationships among breeds. Genotypes from seven additional sheep breeds belonging to International Sheep Genomics Consortium (Additional file 3: Table S3) were included in the analysis to place the Sicilian sheep breeds in a global context. 


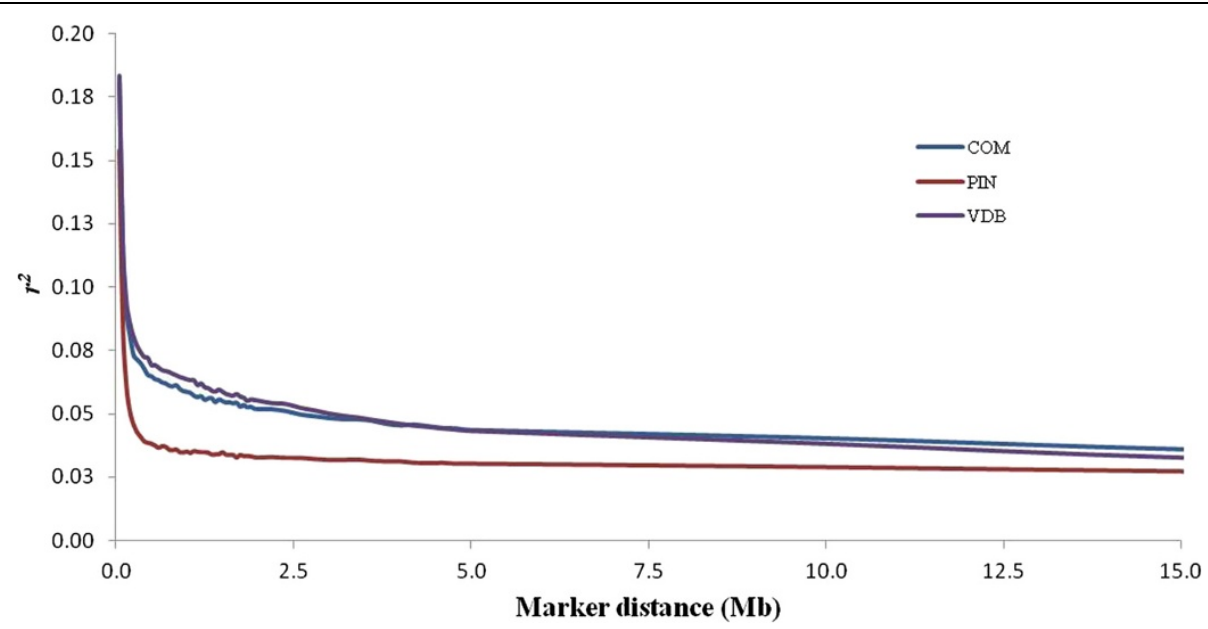

Figure 1 Linkage disequilibrium across the genome as a function of genomic distance. Valle del Belice (VDB), Comisana (COM) and Pinzirita (PIN) breeds.

After data editing, a total of 42,422 SNPs common to 486 individuals from 10 sheep breeds were analyzed. The PCA showed that most sheep breeds formed nonoverlapping clusters and are clearly separated populations, except for Sarda white and Sarda black sheep breeds (Figure 2). Genetic similarity between the two breeds of Sardinia island was in agreement with previous studies [21]. Moreover, the PCA separated the breeds according to their geographic origin, with Italian breeds positioned apart from other European ones.

To explore in detail the relatedness among Sicilian breeds, PCA was performed separately for Valle del Belice, Comisana and Pinzirita breeds. In addition to these autochthonous breeds, Sarda white was considered due to its likely contribution to the phylogenetic origin of Valle del Belice breed. While for Valle del Belice, Pinzirita and Sarda white breeds the two components (PC1 and PC2) clustered animals from the same breed together, the Comisana breed showed two groups (Figure 3). In fact, individuals from Comisana occupied different areas of the cluster, indicating the presence of substructure, and this could evoke concerns about the generation of false positive results when using LD mapping as the only mean to locate genes underlying complex traits [18]. The separation of Comisana individuals in these two sub-populations corresponded approximately to geographical areas of Sicily in which they were collected. Therefore, the genetic structure detected for Comisana sheep breed could be due to
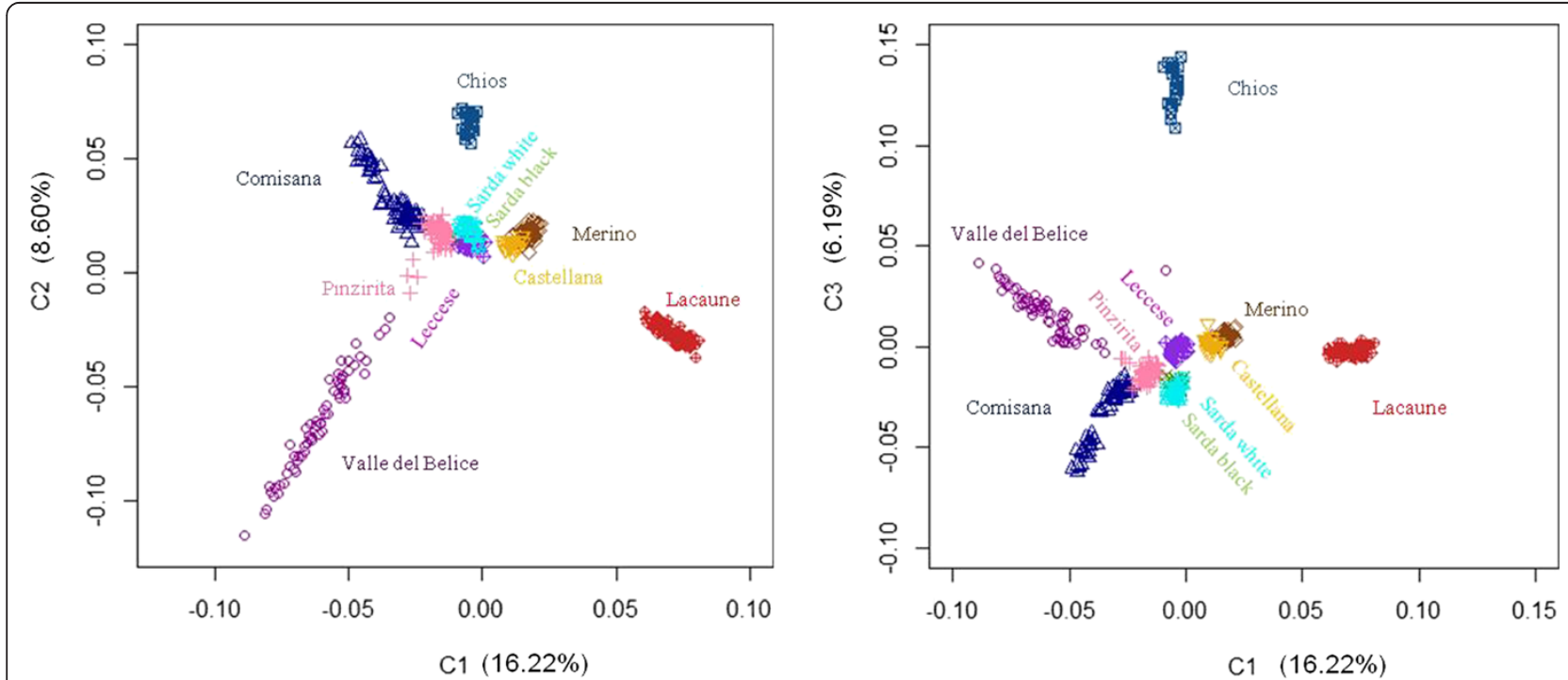

Figure 2 Principal components analysis for the genetic differentiations among all sheep breeds using PC1 and PC2 (left), and PC1 and PC3 (right). 

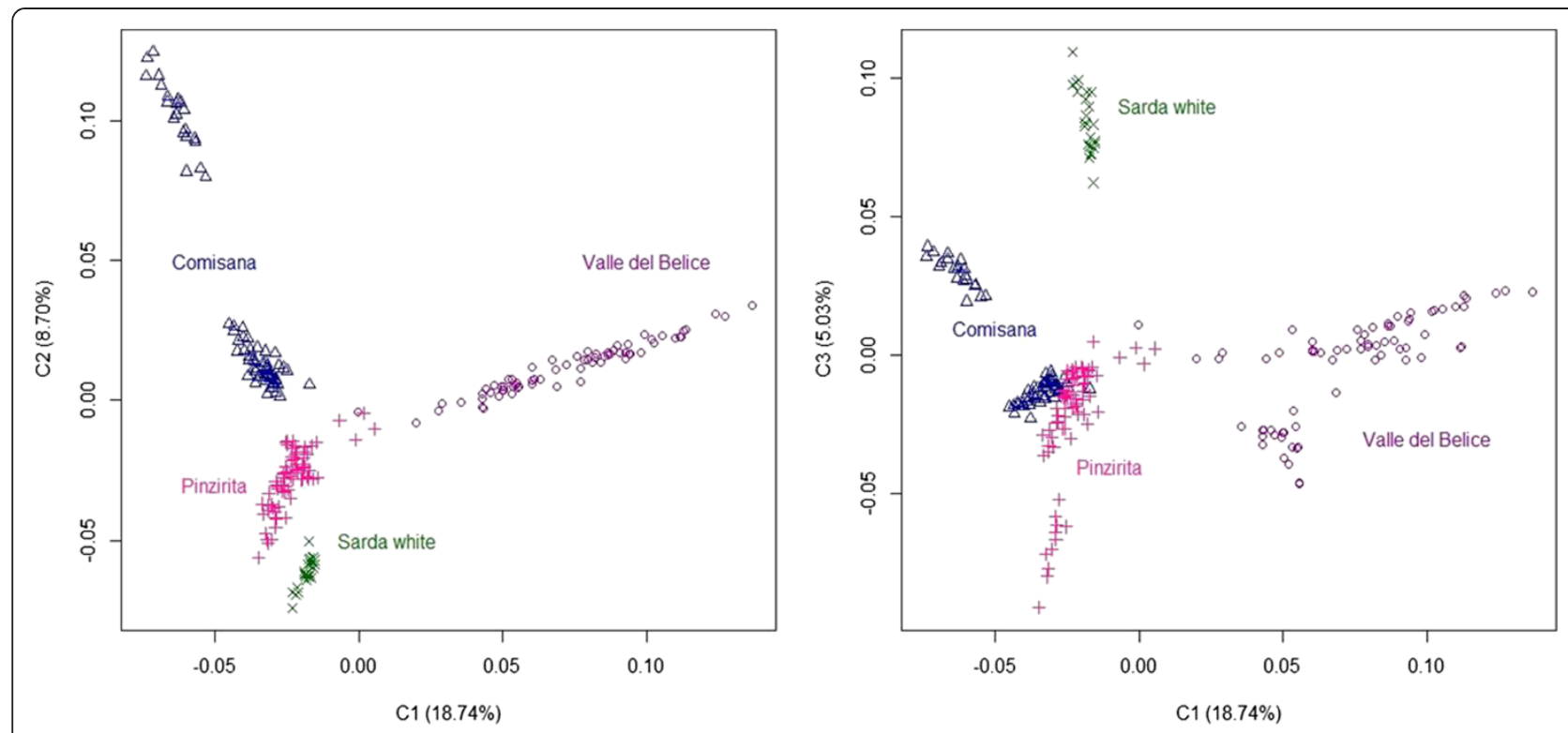

Figure 3 Principal components analysis for the genetic differentiations between Sicilian and Sarda white sheep breeds using PC1 and PC2 (left), and PC1 and PC3 (right).

introgression of genes from other breeds and/or to geographical isolation of some farms for a long time. In fact, cross-breeding is known to be one of the main factors responsible for erosion of local breeds. The presence of substructure was confirmed by combination of $\mathrm{PC} 1$ and $\mathrm{PC} 3$ (Figure 3).

Moreover, some individuals of Comisana breed were positioned near the cluster of Pinzirita breed. The genetic closeness between these two breeds might be explained considering that they are characterized by a common breeding system and geographical husbandry area, which might have led to genetic exchange between them [22]. The relative contribution of SNPs to population assignment was estimated. In fact, high-throughput genotyping tools make it possible to extract interesting genetic information from animal populations that could be applied to identify useful markers for breed allocation, assignment parentage [23], and for tracing geographic origin of animal products [9] as meat and mono-breed cheese. Among the 44,610 SNPs common to the three Sicilian breeds, a subset of 119 SNPs was used to evaluate their ability to cluster individuals belonging to the Sicilian sheep breeds. These SNPs were selected considering their informativeness in pair comparisons that means SNPs with the larger allele frequency differences between pairs of breeds were chosen (fixed alleles in one breed and MAF $>0.25$ in the other ones). In fact, differences in allele frequency provide the basis for assignment of individuals into discrete populations. PCA using this subset of SNPs showed lack of ability to discriminate among the breeds and the presence of overlapping areas, in particular between Comisana and
Pinzirita sheep breeds (Additional file 4: Figure S1). Kijas et al. [7] revealed that while 96 of the most informative SNPs (higher average allelic richness and lower average private allelic richness) were insufficient, analysis using a panel of 384 markers successfully sorted individuals into groups. Our results may be due to close relationships among Sicilian sheep breeds that are genetically connected among them [22,24]. Therefore, the subset of SNPs was not useful to assign individuals into discrete clusters and it was insufficient and inadequate for authentication purposes.

Results from within population substructure through Admixture analysis, considering a range of 2 through 20 potential clusters $(\mathrm{K})$, pointed out that the best fitting number of populations present in the total sample was $\mathrm{K}=12$. A graphic representation of the estimated membership coefficients to the 12 clusters is shown in Figure 4, where model-based clustering partitioned the genome of each sample into a predefined number of components [25]. The first breeds to be differentiated from the others were Lacaune and Valle del Belice $(K=2)$. Other breed-specific clusters were Chios and Merinos $(K=6)$; at $\mathrm{K}=8$ some genomic components appeared to be shared by several breeds, as for example between Comisana and Pinzirita, whereas at $K=10$, each breed tends to have its own distinct cluster but with some differences; in fact, Sicilian breeds showed less distinct clusters than other breeds as Lacaune, Chios and Sarda black. For some breeds, as the two from Sardinia island, the admixture analysis revealed a shared ancestry; in fact these breeds clustered together at high $\mathrm{K}$ value $(\mathrm{K}=10)$. These results reflect geographic proximity and confirmed the findings 


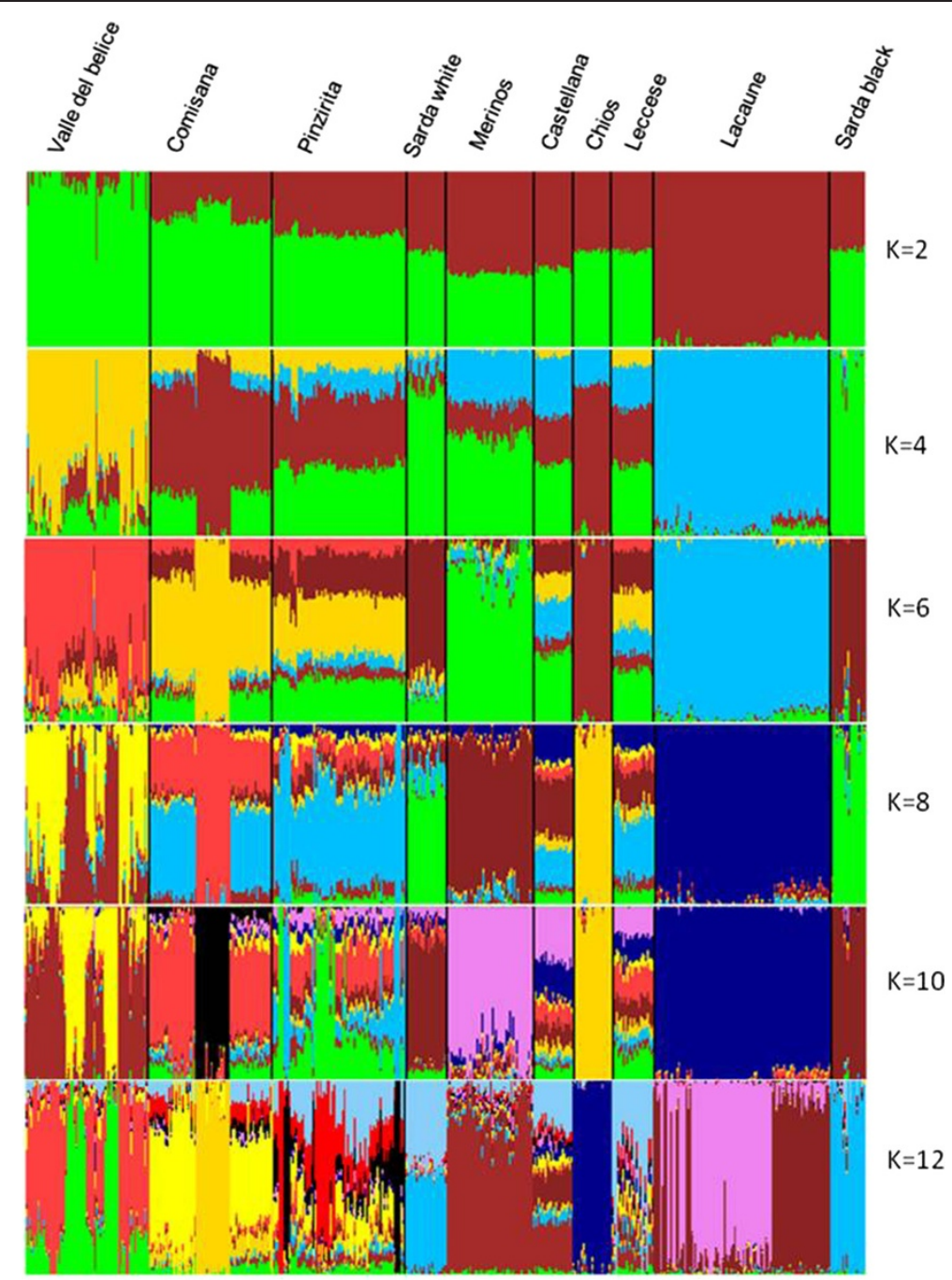

Figure 4 Model based clustering of the estimated membership fractions of individuals of the 10 breeds analyzed in each of the $\mathrm{K}$ inferred clusters, for $K=2$ to $K=12$.

based on the PCA, where, for example, Chios and Lacaune formed separated and differentiated clusters. For the Sicilian breeds, results were in agreement with a previous study conducted on the genetic structure and relationship using microsatellite markers [22] that showed low genetic differentiation and high level of admixture among Valle del Belice, Comisana, and Pinzirita breeds. Moreover, at $\mathrm{K}=10$ the results evidenced again that Comisana is split in two mixed groups. The degree of genetic differentiation between pairs of breeds was showed in Table 2. Considering pairwise $F_{\mathrm{ST}}$ among all populations, Chios was the most divergent breed. The highest values were observed between Chios and both Sarda breeds $\left(F_{\mathrm{ST}}=0.139\right.$ and 0.120 for Sarda black and Sarda white, respectively), while the lowest one was observed between Comisana and Pinzirita breeds $\left(F_{\mathrm{ST}}=0.025\right)$. These results may be explained considering the geographic origin of the breeds: Chios and the two
Sarda breeds belonging to different countries, whereas Comisana and Pinzirita were two breeds reared in the same island. The $F_{\mathrm{ST}}$ value between pairs of breeds was also used to reconstruct the NeighborNet graph (Figure 5), showing some clear clusters and relationships between breeds that originated from the same regions: Sicilian, Sardinian, and European breeds. The shortest branch was observed for Pinzirita breed, while the longest one was found for Chios breed that was the most differentiated and isolated population among the analyzed breeds. Considering only the Sicilian breeds, Valle del Belice showed the longest branch, while Pinzirita the shortest one, according to the results of genetic diversity for these breeds (Table 3). In fact, Kijas et al. [26] in a study on genome-wide analysis of the world's sheep breeds showed short branches for breeds with high heterozygosity, while long branches for breeds with low heterozygosity. The topology of 
Table 2 Genetic differentiation between population pairs measured using $\boldsymbol{F}_{\text {st. }}$

\begin{tabular}{|c|c|c|c|c|c|c|c|c|c|c|}
\hline & PIN & COM & SAB & VDB & $\mathrm{CHI}$ & CAS & MER & SAW & LAC & LEC \\
\hline PIN & 0 & & & & & & & & & \\
\hline COM & 0.025 & 0 & & & & & & & & \\
\hline$S A B$ & 0.065 & 0.080 & 0 & & & & & & & \\
\hline VDB & 0.041 & 0.051 & 0.090 & 0 & & & & & & \\
\hline $\mathrm{CHI}$ & 0.086 & 0.096 & 0.139 & 0.112 & 0 & & & & & \\
\hline CAS & 0.033 & 0.045 & 0.078 & 0.059 & 0.096 & 0 & & & & \\
\hline MER & 0.038 & 0.051 & 0.080 & 0.063 & 0.099 & 0.038 & 0 & & & \\
\hline SAW & 0.046 & 0.060 & 0.059 & 0.071 & 0.120 & 0.060 & 0.061 & 0 & & \\
\hline LAC & 0.041 & 0.054 & 0.083 & 0.066 & 0.103 & 0.044 & 0.048 & 0.064 & 0 & \\
\hline LEC & 0.030 & 0.040 & 0.075 & 0.054 & 0.095 & 0.038 & 0.043 & 0.054 & 0.047 & 0 \\
\hline
\end{tabular}

$(\mathrm{PIN}=$ Pinzirita, $\mathrm{COM}=$ Comisana, $\mathrm{SAB}=$ Sarda black, VDB = Valle del Belice, $\mathrm{CHI}=$ Chios, CAS = Castellana, MER = Merino, SAW $=$ Sarda white, LAC $=$ Lacaune, LEC $=$ Leccese).

the NeighborNet constructed with $F_{\mathrm{ST}}$ distances between breeds perfectly coincides with PCA (Figure 2). NeighborNet graph was also depicted considering the substructure present in Comisana breed (Additional file 5: Figure S2). The figure showed that the two subpopulations originated from the same branch and displayed a very close relationship, which was expected considering that belonging to the same breed. The reticulations towards the extremity of the graphs indicated increasing genetic relatedness between breeds. In fact, NeighborNet provide a robust framework for inferring and investigating phylogenetic networks.

Understanding the relationships among and within populations is an important step to establish conservation priorities and strategies. Consistency of results among the several used approaches (PCA, Bayesian clustering, $F_{\mathrm{ST}}$, Neighbor networks) supported the robustness of our conclusions.

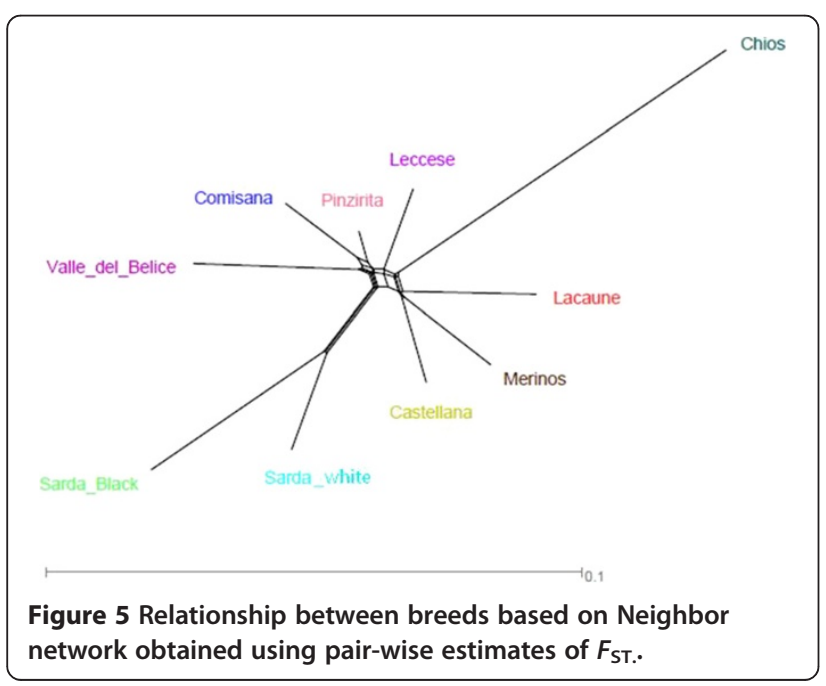

Estimates of the genetic diversity in Sicilian sheep breeds The basic genetic diversity indices within breed were used to compare levels of heterogeneity between Sicilian breeds. Overall average MAF was $0.290 \pm 0.003$ for Valle del Belice, $0.294 \pm 0.004$ for Comisana, and $0.301 \pm 0.005$ for Pinzirita sheep breeds (Table 3). These values were in agreement with those reported by Kijas et al. [26] in a study on genome-wide analysis of the world's sheep breeds for the European-derived populations. Moreover, the distribution of MAF of these SNPs was approximately uniform over the genome in all breeds (Additional file 1: Table S1). Pinzirita breed displayed the highest gene diversity $(\mathrm{He}=0.390 \pm 0.108)$, whereas the lowest value was found in Valle del Belice breed $(\mathrm{He}=0.379 \pm 0.155)$ (Table 3). These genetic diversity estimates can be compared with those reported by other authors for Southern and Mediterranean European sheep breeds [26]. Similar results for genetic diversity ( $\mathrm{Ho}, \mathrm{He}$, and MAF) were reported for Sarda sheep breed [14]. We obtained some negative values for inbreeding coefficient $F$, which corresponded to animals with lower homozygosity than the average population. The highest $F$, calculated for each individual based upon observed and expected heterozygosity, was found in Valle del Belice breed $(0.055 \pm 0.150)$, whereas the lowest value in Pinzirita breed $(0.016 \pm 0.042)$. In Valle del Belice breed, the high level of genetic differentiation within breed, with individuals spread out on PC1 (Figure 3), and heterogeneous cluster in Admixture analysis (Figure 4), but at the same time the highest inbreeding coefficient (Table 3), suggested that the breed had a wide genetic base with inbred individuals belonging to the same flock. Rosa et al. [27] in a study on parentage verification of Valle del Belice breed, reported different values of inbreeding per flock that ranged from 0.017 to 0.165 . In fact, in Sicilian farming system natural mating is the common practice, the exchange of rams among flocks is quite unusual, and mating with close relatives can be quite 
Table 3 Estimates of genetic diversity indices for Sicilian sheep breeds

\begin{tabular}{|c|c|c|c|c|c|}
\hline Breeds & MAF \pm s.d. & Ho \pm s.d. & $\mathrm{He} \pm$ s.d. & $F \pm$ s.d. & $\mathrm{N}_{\mathrm{e}}$ \\
\hline Valle del Belice & $0.290 \pm 0.003$ & $0.364 \pm 0.126$ & $0.379 \pm 0.155$ & $0.055 \pm 0.150$ & 369 \\
\hline Comisana & $0.294 \pm 0.004$ & $0.382 \pm 0.129$ & $0.382 \pm 0.114$ & $0.025 \pm 0.031$ & 400 \\
\hline Pinzirita & $0.301 \pm 0.005$ & $0.388 \pm 0.122$ & $0.390 \pm 0.108$ & $0.016 \pm 0.042$ & 685 \\
\hline
\end{tabular}

Average minor allele frequency (MAF), observed heterozygosity (Ho), expected heterozygosity (He), inbreeding coefficient (F) and standard deviation (s.d.), and effective population size $\left(\mathrm{N}_{\mathrm{e}}\right)$.

frequent; this led to high level of inbreeding. Analogous results for Valle del Belice breed were reported by Tolone et al. [22]. Therefore, the results (high level of genetic differentiation and high inbreeding coefficient) may be explained considering reduced or absent gene flow between different flocks. It should be noted that Valle del Belice was the most homogeneous breed at the lowest $\mathrm{K}$ values, according with higher inbreeding and lower heterozygosity. Moreover, the low level of inbreeding and high genetic diversity in Pinzirita sheep breed reflected the short extent of LD. Average values of LD for $1 \mathrm{Mb}$ bins smoothed with a $50 \mathrm{~kb}$ step size were used to estimate the effective population size $\left(\mathrm{N}_{\mathrm{e}}\right)$. In fact, LD is affected by population history and demography, representing an important tool to be applied to genetic population. Effective population size $\left(\mathrm{N}_{\mathrm{e}}\right)$ is a general indicator of the risk of genetic erosion, contains relevant information for the monitoring of the genetic diversity, and helps to explain how populations evolved [28]. The highest value of $\mathrm{N}_{\mathrm{e}}$, estimated 50 generations ago, was observed for Pinzirita breed $\left(\mathrm{N}_{\mathrm{e}}=685\right)$, whereas the lowest one was observed for Valle del Belice breed $\left(\mathrm{N}_{\mathrm{e}}=369\right)$ (Table 3). In fact, high $\mathrm{N}_{\mathrm{e}}$ is accompanied with high genetic diversity and low values of LD. Moreover, it is important to remind that LD measures, and therefore $\mathrm{N}_{\mathrm{e}}$, are linked with selection intensity and mating systems. Indeed, high selection pressure and the use of artificial insemination are the main reasons for low $\mathrm{N}_{e}$ values. Ciani et al. [21] in a study on Italian sheep breeds reported similar results of $\mathrm{N}_{\mathrm{e}}$ in Sicilia sheep breeds with 638, 571, and 340 individuals for Pinzirita, Comisana, and Valle del Belice breeds, respectively. Moreover, GarcíaGámez et al. [1] in Churra sheep breed reported $\mathrm{N}_{\mathrm{e}}$ estimated 50 generation ago of 467 individuals. Our results were consistent with those reported for most European sheep breeds [26] that showed high $\mathrm{N}_{\mathrm{e}}$. Managing the $\mathrm{N}_{\mathrm{e}}$ and $F$ provides a general framework to control loss of variability avoiding or alleviating reductions in viability and fertility; i.e., inbreeding depression [29].

\section{Conclusions}

This study reported for the first time estimates of linkage disequilibrium, genetic diversity and population structure from a genome-wide perspective in Sicilian dairy sheep breeds. Knowledge concerning the behavior of LD is important for performing genomic selection and genome wide association analysis. LD declined as a function of distance and average $r^{2}$ was lower than value observed in other sheep breeds. Results indicated that Sicilian sheep breeds formed non-overlapping clusters, with low genetic differentiation and high level of admixture, and that the Comisana breed does not constitute a homogenous population. The different approaches (PCA analysis, Bayesian clustering, $F_{\mathrm{ST}}$, NeighborNet) used to assess relationships among breeds showed high degree of congruence and supported the robustness of conclusions. Analysis of genetic diversity indicated high genetic variability and low inbreeding. The identification of genetic relationship and gene flow among livestock breeds/populations is important for breeders and conservationists. In order to maintain the existing genetic diversity, breeding strategies aiming at maintaining effective population size, minimizing inbreeding and genetic drift should be implemented for the Sicilian breeds. The information generated from this study can have important implications for the design and applications of association studies as well as for the development of conservation and/or selection breeding programs.

\section{Methods}

\section{DNA sampling and genotyping}

A total of 221 unrelated animals were collected from several farms in different areas of Sicily, to capture a representative sample of within breed genetic diversity, and were used for the analysis.

The procedures involving animal sample collection followed the recommendation of directive 2010/63/EU. Number of animal sampled per flock ranged from 5 to 10 . Samples consisted of 72 Valle del Belice, 72 Comisana and 77 Pinzirita individuals. For these sheep breeds, pedigree data were not available. About $10 \mathrm{ml}$ of blood was collected from jugular vein using tubes with EDTA as anticoagulant. Genomic DNA was extracted from buffy coats of nucleated cells using salting out method [30]. The concentration of extracted DNA was assessed with NanoDrop ND-1000 spectrophotometer (NanoDrop Technologies, Wilmington, DE).

All animals were genotyped for 54,241 SNPs, using the Illumina OvineSNP50K Genotyping BeadChip following standard operating procedures recommended by the manufacturer. Genotyping was performed by Dipartimento Scienze Agrarie e Forestali, University of Palermo. Raw signal intensities were converted into genotype calls using the Illumina GenomeStudio Genotyping Module 
v1.0 software (Illumina Inc., San Diego, CA) by applying a no-call threshold of 0.15 . Genotyping data were initially tested for quality using the same software. Markers in each breed were filtered to exclude loci assigned to unmapped contigs. Therefore, only SNPs located on autosomes were considered in further analyses. Moreover, quality control included: Call Frequency (proportion of samples with genotype at each locus) $\geq 0.95$, minor allele frequency $(\mathrm{MAF}) \geq 0.05$, and Hardy-Weinberg Equilibrium (HWE) $P$-value $>0.001$. SNPs that did not satisfy these quality criteria were discarded.

\section{Linkage Disequilibrium}

A standard descriptive Linkage Disequilibrium (LD) parameter, the squared correlation coefficient of allele frequencies at pair of loci $\left(r^{2}\right)$, was used as measure. Pairwise LD between adjacent SNPs was calculated on each chromosome using PLINK [31]. Moreover, $r^{2}$ was estimated for all pairwise combinations of SNPs using LD plot function in Haploview v4.2 software [32], exporting data to text files. For each chromosome, pairwise $r^{2}$ was calculated for SNPs between 0 and $50 \mathrm{Mb}$ apart. To visualize the LD pattern per chromosome, $r^{2}$ values were stacked and plotted as a function of inter-marker distance categories. Average $r^{2}$ for SNP pairs in each interval was estimated as the arithmetic mean of all $r^{2}$.

\section{Analysis of genetic diversity and population structure among breeds}

In order to understand the genetic relationship among Sicilian breeds, we performed different analyses. Genotypes from others 7 sheep breeds belonging to International Sheep Genomics Consortium (Additional file 2: Table S2), were included in these analyses. Genotyping data from Sicilian and other breeds were filtered with the same quality criteria reported above. Unlinked SNPs were selected using -indep option of the PLINK [31], to reduce the impact of the SNP ascertainment bias phenomenon, with the following parameters: 50 SNPs per window, a shift of 5 SNPs between windows, and a variation inflation factor's threshold of 2 .

First, the average proportion of alleles shared between animals $\left(A_{s}\right)$ was calculated as IBS $2+0.5 \% \mathrm{IBS} 1 / \mathrm{N}$, where IBS1 and IBS2 are the number of loci that share either one or two alleles identical by state (IBS), respectively, and $\mathrm{N}$ is the number of loci tested. Genetic distance $(D)$ was calculated as $1-A_{s}$. These values were calculated using PLINK [31] through the use of commands -cluster and-distance-matrix. PCA of $D$ matrix was performed using the multidimensional scaling (MDS) algorithm of pairwise genetic distance implemented in PLINK [31]. It should be noted that when MDS is applied to $D$ matrix, it is numerically identical to PCA [31]. The graphical representation was depicted using the statistical $R$ software (R Development Core Team) with RColorBrewer package.

Extents of population substructure were evaluated through the model-based clustering algorithm implemented in the software Admixture [33]. The most probable number of populations in the dataset $(\mathrm{K})$ was estimated using the default (5-fold) Admixture's cross-validation procedure, by which estimated prediction errors are obtained, for each $\mathrm{K}$ value, by adopting a kind of 'leave one-out' approach. $K$ value that minimizes the estimated prediction errors is then assumed to be the most suitable. Graphical representation was visualized using the statistical $R$ software (R Development Core Team).

Genepop [34] software was used to estimate population relatedness using pair-wise estimates of $F_{\mathrm{ST}}$ among all breeds. Neighbor networks were constructed from pair-wise estimates of $F_{\mathrm{ST}}$ using Splitstree [35].

\section{Estimates of the genetic diversity in Sicilian sheep breeds} PLINK [31] was also used to estimate basic genetic diversity indices, including observed and expected heterozygosity (Ho and $\mathrm{He}$, respectively), average MAF and coefficient of inbreeding $(F)$ for Sicilian sheep breeds. Files used for basic diversity indices ( $\mathrm{Ho}, \mathrm{He}$ and $F$ ) were pruned in PLINK considering 50 SNPs per windows, a shift of 10 SNPs between windows and variation inflation factor's threshold of 1.5. Moreover, effective population sizes $\left(\mathrm{N}_{\mathrm{e}}\right)$ were calculated as $\mathrm{N}_{\mathrm{e}}=(1 / 4 c)^{*}\left(1 / r^{2}-1\right)$ [36], where $r^{2}$ is the value of LD and $c$ is the marker distance in Morgans between SNPs.

\section{Additional files}

Additional file 1: Table S1. Average MAF (Minor Allele Frequency) per chromosome (OAR) and number of SNPs $\left(\mathrm{N}^{\circ}\right)$ that passed quality control in the Sicilian sheep breeds. Valle del Belice (VDB), Comisana (COM), and Pinzirita (PIN) sheep breeds.

Additional file 2: Table S2. Mean Linkage Disequilibrium $\left(r^{2}\right)$ among SNPs over different map distances in Sicilian sheep breeds. Valle del Belice (VDB), Comisana (COM), and Pinzirita (PIN) sheep breeds.

Additional file 3: Table S3. Number of sampled animals from seven additional sheep breeds.

Additional file 4: Figure S1. Principal components analysis between Sicilian breeds using marker panel contained 119 SNPs. Valle del Belice (VDB), Comisana (COM), and Pinzirita (PIN) breeds.

Additional file 5: Figure S2. Neighbor network obtained using pairwise estimates of $F_{S T}$ between breeds, considering the sub-populations in Comisana breed.

\section{Competing interests}

The authors have no competing to declare.

\section{Authors' contributions}

MS, SMT and PB conceived and designed the experiments. MS drafted the manuscript. DGR and TL carried out DNA extraction, purification and analyses. MS, SMT, DGR and TM analyzed the data and performed the statistical analysis. ISGC provided genotypes of seven additional sheep breeds. All authors read and approved the final manuscript. 


\section{Acknowledgements}

This research was financed by PON02_00451_3133441,

CUP: B61C1200076005 funded by MIUR.

Received: 30 May 2014 Accepted: 29 September 2014

Published online: 10 October 2014

\section{References}

1. García-Gámez E, Sahana G, Gutiérrez-Gil B, Arranz JJ: Linkage disequilibrium and inbreeding estimation in Spanish Churra sheep. BMC Genet 2012, 13:43.

2. Karlsson EK, Baranowska I, Wade CM, Salmon Hillbertz NHC, Zody MC, Anderson N, Biagi TM, Patterson N, Pielberg GR, Kulbokas EJ, Comstock KE, Keller ET, Mesirov JP, von Euler H, Kampe O, Hedhammar A, Lander ES, Andersson G, Andersson $\mathrm{L}$, Lindblad-Toh $\mathrm{K}$ : Efficient mapping of mendelian traits in dogs through genome-wide association. Nat Genet 2007, 39:1321-1328.

3. Meuwissen THE, Goddard ME: Fine mapping of quantitative trait loci using linkage disequilibrium with closely linked marker loci. Genetics 2000, 155:421-430.

4. Meuwissen THE, Hayes BJ, Goddard ME: Prediction of total genetic value using genome wide dense marker maps. Genetics 2001, 155:945-959.

5. Hayes BJ, Visscher PM, McPartlan HC, Goddard ME: Novel multilocus measure of linkage disequilibrium to estimate past effective population size. Genome Res 2003, 13:635-643.

6. Meadows JRS, Chan EKF, Kijas JW: Linkage disequilibrium compared between five populations of domestic sheep. BMC Genet 2008, 9:61.

7. Kijas JW, Townley D, Dalrymple BP, Heaton MP, Maddox JF, McGrath A, Wilson P, Ingersoll RG, McCulloch R, McWilliam S, Tang D, McEwan J, Cockett N, Hutton Oddy V, Nicholas FW, Raadsma H, International Sheep Genomics Consortium: A Genome Wide Survey of SNP Variation Reveals the Genetic Structure of Sheep Breeds. PLoS ONE 2009, 4(3):e4668.

8. Boettcher PJ, Tixier-Boichard M, Toro MA, Simianer H, Eding H, Gandini G, Joost S, Garcia D, Colli L, Ajmone-Marsan P, GLOBALDIV Consortium: Objectives, criteria and methods for using molecular genetic data in priority setting for conservation of animal genetic resources. Anim Genet 2010, 41:64-77.

9. Li MH, Strandén I, Tiirikka T, Sevón-Aimonen ML, Kantanen J: A comparison of approaches to estimate the inbreeding coefficient and pairwise relatedness using genomic and pedigree data in a sheep population. PLOS ONE 2011, 6:e26256.

10. Scintu MF, Piredda G: Typicity and biodiversity of goat and sheep milk products. Small Rumin Res 2007, 68:221-231.

11. Zhao H, Nettleton D, Dekkers JCM: Evaluation of linkage disequilibrium measures between multi-allelic markers as predictors of linkage disequilibrium between single nucleotide polymorphisms. Genet Res 2007, 89:1-6.

12. Khatkar MS, Nicholas FW, Collins AR, Zenger KR, Cavanagh JA, Berris W, Schnabel RD, Taylor JF, Raadsma HW: Extent of genome-wide linkage disequilibrium in Australian Holstein-Friesian cattle based on a highdensity SNP panel. BMC Genomics 2008, 9:187.

13. Qanbari S, Pimentel EC, Tetens J, Thaller G, Lichtner P, Sharifi AR, Simianer H: The pattern of linkage disequilibrium in German Holstein cattle. Anim Genet 2010, 41:346-356.

14. Usai MG, Sechi T, Salaris S, Cubeddu T, Roggio T, Casu S, Carta A: Analysis of a representative sample of Sarda breed artificial insemination rams with the OvineSNP50 BeadChip. In Proceedings of 37th International Committee for Animal Recording (ICAR) Biennial Session: 31st May-4th June 2010; Riga, Latvia. Edited by Skujina E, Galvanoska E, Leray O, Mosconi C.: 2010:7-10.

15. Miller JM, Poissant J, Kijas JW, Coltman DW, International Sheep Genomics Consortium: A genome-wide set of SNP detects population substructure and long range linkage disequilibrium in wild sheep. Mol Ecol Resour 2010, 11:314-322.

16. Veroneze R, Lopes PS, Guimaraes SEF, Silva FF, Lopes MS, Harlizius B, Knol EF: Linkage disequilibrium and haplotype block structure in six commercial pig lines. J Anim Sci 2013, 91(8):3493-3501.

17. Uimari P, Tapio M: Extent of linkage disequilibrium and effective population size in Finnish Landrace and Finnish Yorkshire pig breeds. J Anim Sci 2011, 89(3):609-614.

18. Beghain J, Boitard S, Weiss B, Boussaha M, Gut I, Rocha D: Genome wide linkage disequilibrium in the Blonde d'Aquitaine cattle breed. J Anim Breed Genet 2013, 130(4):294-302.
19. Corbin LJ, Blott SC, Swinburne JE, Vaudin M, Bishop SC, Woolliams JA: Linkage disequilibrium and historical effective population size in the Thoroughbred horse. Anim Genet 2010, 41(2):8-15.

20. McCue ME, Bannasch DL, Petersen JL, Gurr J, Bailey E, Binns MM, Distl O, Guérin G, Hasegawa T, Hill EW, Leeb T, Lindgren G, Penedo CMT, Røed KH, Ryder OA, Swinburne JE, Tozaki T, Valberg SJ, Mark Vaudin M, Lindblad-Toh K, Wade CM, Mickelson JR: A High Density SNP Array for the Domestic Horse and Extant Perissodactyla: Utility for Association Mapping, Genetic Diversity, and Phylogeny Studies. PLoS Genet 2012, 8(1):e1002451.

21. Ciani E, Crepaldi P, Nicoloso L, Lasagna E, Sarti FM, Moioli B, Napolitano F, Carta A, Usai G, D'Andrea M, Marletta D, Ciampolini R, Riggio V, Occidente M, Matassino D, Kompan D, Modesto P, Macciotta N, Ajmone-Marsan P, Pilla F: Genome-wide analysis of Italian sheep diversity reveals a strong geographic pattern and cryptic relationships between breeds. Anim Genet 2013, 45:256-266.

22. Tolone M, Mastrangelo S, Rosa AJM, Portolano B: Genetic diversity and population structure of Sicilian sheep breeds using microsatellite markers. Small Rumin Res 2012, 102:18-25.

23. Fisher PJ, Malthus B, Walker MC, Corbett G, Spelman RJ: The number of single nucleotide polymorphisms and on-farm data required for wholeherd parentage testing in dairy cattle herds. J Dairy Sci 2009, 92:369-374.

24. Mastrangelo S, Sardina MT, Riggio V, Portolano B: Study of polymorphisms in the promoter region of ovine b-lactoglobulin gene and phylogenetic analysis among the Valle del Belice breed and other sheep breeds considered as ancestors. Mol Biol Rep 2012, 39:745-751.

25. Pritchard JK, Stephens M, Donnelly P: Inference of population structure using multilocus genotype data. Genetics 2000, 155:945-959.

26. Kijas JW, Lenstra JA, Hayes B, Boitard S, Porto Neto LR, Cristobal MS, Servin B, McCulloch R, Whan V, Gietzen K, Paiva S, Barendse W, Ciani E, Raadsma H, McEwan J, Dalrymple B, International Sheep Genomics Consortium: Genome-Wide Analysis of the world's sheep breeds reveals high levels of historic mixture and strong recent selection. PLos Biol 2012, 10(2):e1001258,

27. Rosa AJM, Sardina MT, Mastrangelo S, Tolone M, Portolano B: Parentage verification of Valle del Belice dairy sheep using multiplex microsatellite panel. Small Rumin Res 2013, 113:62-65.

28. Tenesa A, Navarro P, Hayes BJ, Duffy DL, Clarke GM, Goddard ME, Visscher PM: Recent human effective population size estimated from linkage disequilibrium. Genome Res 2007, 17:520-526.

29. Villanueva B, Pong-Wong R, Woolliams JA, Avendaño S: Managing genetic resources in selected and conserved populations. In Farm Animal Genetic Resources Edited by Simm G, Villanueva B, Sinclair KD, Townsend S. Nottingham: Nottingham University Press; 2004:113-132.

30. Miller SA, Dykes DD, Polesky HF: A simple salting out procedure for extracting DNA from human nucleated cells. Nucleic Acids Res 1988, 16:1215.

31. Purcell S, Neale B, Todd-Brown K, Thomas L, Ferreira MA, Bender D, Maller J, Sklar P, De Bakker PI, Daly MJ, Sham PC: PLINK: A tool set for wholegenome association and population-based linkage analyses. Am J Hum Genet 2007, 81:559-575.

32. Barrett JC, Fry B, Maller J, Daly MJ: Haploview: analysis and visualization of LD and haplotype maps. Bioinformatics 2005, 21:263-265.

33. Alexander DH, Lange $\mathrm{K}$ : Enhancements to the ADMIXTURE algorithm for individual ancestry estimation. BMC Bioinformatics 2011, 12:246.

34. Raymond M, Rousset F: GENEPOP population genetics software for exact tests and ecumenicism. J Hered 1995, 86:248-249.

35. Huson DH, Bryant D: Application of phylogenetic networks in evolutionary studies. Mol Biol Evol 2006, 23:254-267.

36. Sved JA: Linkage disequilibrium of chromosome segments. Theor Popul Biol 1971, 141:125-141.

doi:10.1186/s12863-014-0108-5

Cite this article as: Mastrangelo et al:: Genome wide linkage disequilibrium and genetic structure in Sicilian dairy sheep breeds. BMC Genetics 2014 15:108. 